Research Paper

\title{
SZRD1 is a Novel Protein that Functions as a Potential Tumor Suppressor in Cervical Cancer
}

\author{
Ning Zhao ${ }^{1,2}$, Guoying Zhang,1,2, Minwei He ${ }^{1,2}$, He Huang,1,2, Lulu Cao ${ }^{1,2}$, Ang Yin ${ }^{1,2}$, Pingzhang Wang ${ }^{1,2}$, Lu \\ Wang ${ }^{1,2} \square$ \\ 1. Center for Human Disease Genomics, Department of Immunology, School of Basic Medical Sciences, Health Science Center, Peking University, Beijing \\ 100191, P. R. China \\ 2. Key Laboratory of Medical Immunology, Ministry of Health, School of Basic Medical Science, Peking University, Beijing 100191, P. R. China \\ $\triangle$ Corresponding author: Dr. Lu Wang, Center for Human Disease Genomics, Peking University, 38 Xueyuan Road, Beijing 100191, P. R. China. E-MAIL: \\ wanglu@bjmu.edu.cn; TEL: +86-10-82802710-5037; FAX: +86-10-82801149 \\ (c) Ivyspring International Publisher. This is an open access article distributed under the terms of the Creative Commons Attribution (CC BY-NC) license \\ (https://creativecommons.org/licenses/by-nc/4.0/). See http://ivyspring.com/terms for full terms and conditions.
}

Received: 2016.12.18; Accepted: 2017.04.10; Published: 2017.07.14

\begin{abstract}
SZRDI is a novel gene screened out by high-throughput platform, and so far there exists no systematic function reports. The purpose of our study is to discover the function and mechanism of this novel human gene. Bioinformatics analysis indicates that SZRDI is a highly conserved intracellular protein. After overexpression of SZRDI, we found that SZRDI could arrest the cell cycle in G2 phase and play a role in inhibiting cell proliferation and inducing apoptosis. In contrast, after knockdown of endogenous SZRDI, we concluded that it could promote cell proliferation. The mechanism investigations showed that overexpression of SZRDI could downregulate the phosphorylation of ERK1/2, AKT, STAT3 and downstream signaling molecules, and then arrest the cells in G2 phase by upregulating P21. Tissue microarray analysis showed that the expression of SZRDI was downregulated in cervical squamous cell carcinomas compared with normal squamous epithelium, and the ratio of downregulation correlated with the stage of the cancer. Overall, we clarified the function of this novel protein SZRDI, which indicated it may be a potential novel tumor suppressor in cervical cancer.
\end{abstract}

Key words: SZRD1; novel protein; proliferation; apoptosis; G2 arrest; cervical cancer

\section{Introduction}

In the post-genome era, addressing gene function is an important and arduous task. Currently, for most of human encoding genes, their functions are still unclear. With the continuous research of human novel genes, many oncogenes have been found in our body such as C-myc [1] and STAT3 [2] etc. There are also many tumor suppressor genes such as p53 [3] and PTEN [4] etc. On the basis of these studies, great progress has been made in cancer diagnosis and treatment, but in general, the common cancer morbidity and mortality remain high. This requires us to further explore the world of our body, and make clear the mechanism of tumorigenesis and development. Then researches on the functionunknown novel proteins of our body, especially those closely related to cancers, are urgently needed.
To study the function of human novel encoding genes, our laboratory has established various high-throughput platforms to screen the functional clues and further investigate their functions, such as NSA2 [5], TMEM174 [6] and TM9SF1 [7], which are associated with cellular proliferation, death and autophagy, respectively. SUZ RNA binding domain containing 1(SZRD1), also known as C1orf144, is a novel gene identified in our laboratory by using high-throughput screening and currently given no function reports. SZRD1 has three prominent blocks of conservation, which are respectively N-terminal domain, SUZ domain and SUZ-C domain. The SUZ domain is a conserved RNA-binding domain found in eukaryotes and enriched in positive-charged amino acids. The SUZ-C domain is a conserved motif found 
in one or more copies in several RNA-binding proteins. It is always found at the $C$ terminus of the protein and appears to be required for localization of the protein to specific subcellular structures. The SUZ and SUZ-C domain were first characterized in the C.elegans protein SZY-20 which localizes to the centrosome. SZY-20 also contains SUZ and SUZ-C domain. As a putative RNA-binding protein, it can negatively regulate the centrosome duplication and plays a critical role in limiting centrosome size in C. elegans [8], These clues suggest SZRD1 may be a cell cycle related protein.

Cell cycle progression is related to the coordinated activation of cyclin dependent kinase (CDK)/cyclin complexes [9]. The G2/M checkpoint is a significant target for anticancer drugs. P21 is a potent, tight-binding inhibitor of CDKs and increases phosphorylation of cell cycle kinase Cdc2 (CDK1) in p53-dependent sustained G2 arrest [10]. P21 gives the cells sufficient time to repair the damaged gene by blocking the cell cycle, so as to maintain the genetic stability of the cells. If gene repair fails, cells will be induced apoptosis [11]. Apoptosis is a form of programmed cell death characterized by morphological and biochemical changes. In terms of morphological changes, apoptosis can be exemplified by nuclear condensation, and plasma membrane blebbing. Biochemical changes include mitochondrial outer membrane permeabilization and activation of caspases (caspase-3) [12,13]. By blocking the cancer in the G2 phase and inducing apoptosis, it can make a therapeutic effect on cancer. The G2 checkpoint is therefore an attractive target for cancer specific sensitization to DNA-damaging chemotherapy and radiotherapy [14].

Previous studies have shown that SZRD1 may be a putative MAPK activating protein [15]. To analyze the MAPK signaling pathway, they have carried out a large-scale identification and characterization of human genes that activates MARK signaling pathways. By using luciferase reporter gene assay, SZRD1 was identified being involved in the MAPK pathways. Despite the relatively well-identified functions of SUZ domain and signaling pathway, there isn't any research directly showing the function of the novel protein SZRD1. The biological function and regulation mechanism of SZRD1 need to be further studied.

In our study, the function of SZRD1 was detected in vitro. The results clearly indicated that SZRD1 functioned as a growth suppressor via downregulating the phosphorylation of ERK1/2, AKT and STAT3, further resulted in subsequent induction of G2/M cell cycle arrest and apoptosis by inducing the expression of P21. Bioinformatics were performed, and showed a downward trend of SZRD1 expression in many tumors. To further detect the expression patterns of SZRD1, 31 paired cervical cancer tissues were detected using immunohistochemistry and we found that SZRD1 expression was frequently downregulated in cervical cancer tissues, and negatively correlated with malignant phenotypes of cervical cancer. These results all support SZRD1 as a tumor suppressor in cervical cancer.

\section{Materials and methods}

\section{Cell culture and reagents}

HeLa, HEK293T and K562 cells were obtained from the American Type Culture Collection and routinely cultured in RPMI-1640 (HyClone, Logan, UT) supplemented $10 \%$ fetal bovine serum (HyClone, Logan, UT, USA), $100 \mathrm{mg} / \mathrm{mL}$ penicillin-streptomycin in a humidified $5 \% \mathrm{CO} 2$ at $37^{\circ} \mathrm{C}$. All other cell lines used for RT-PCR were maintained in our laboratory. The siRNA of SZRD1 was synthesized by Gene Pharma (Shanghai, China). The si-SZRD1 sequences were used: si-SZRD1-963, 5'GCCAGCAAU AACAGUUUAUTT3' (sense), 5'AUAAACUGUU AUUGCUGGCTT3' (antisense). LipofectamineTM 3000 were purchased from Life Technologies (USA). All transfections were performed according to the instructions. Antibodies against ERK1/2 and phospho-ERK1/2(Thr202/Tyr204), AKT(pan) and phospho-AKT(Ser473), STAT3 and phospho-STAT3 (Tyr705), phospho-p70 (Thr389), phospho-mTOR (Ser2448), RAS, RAF1, P21 and caspase-3 were purchased from Cell Signaling technology (USA). Rabbit anti-SZRD1 polyclonal antibodies were prepared by using human SZRD1 polypeptide and purified in our laboratory.

\section{Bioinformatic analysis}

In addition, 228 samples of human HEK293 cells from the sample platform were also analysed to calculate the correlated genes to SZRD1. The expressional correlation analysis was described previously in $R$ software environment [16]. Genes with the Pearson's coefficients larger than or equal to 0.7 was identified, and functional enrichment analysis was performed by the DAVID web server (http:// david.ncifcrf.gov) [17].

Cancer data from the Affymetrix human genome U133 plus 2.0 platform were collected from the GEO database (http://www.ncbi.nlm.nih.gov/geo/). All of the array data about cancers were processed using previously described data processing and microarray analysis methods $[16,18]$, a TumourProfile database (http://tumour.bjmu.edu.cn/, unpublished) has been developed in our laboratory to analyze the difference of expressed genes in tumors. The expression profile 
of SZRD1 in a variety of cancers and the corresponding control (normal or non-tumor) tissues were collected in this database, and the average rank scores (ARS) represented expression levels of SZRD1, which was described previously [18].

\section{Reverse transcription PCR and real-time quantitative PCR}

The cDNA libraries of tumor cells were prepared in our laboratory and the cDNA libraries of normal human tissue were purchased from Clontech (Mountain View, CA, USA). Total RNA was extracted using TRIzol reagent (Life Technologies, USA). To generate cDNA, reverse transcription was performed using the cDNA Synthesis Kit (Thermo, USA) according to the manufacturer's instructions. Normalization for real-time PCR was performed using the "Power SYBR Green PCR Master Mix" (Applied Biosystems, Forster City, CA, USA). The specific forward and reverse primer were listed as follows: SZRD1， 5'-GGAAATAGACAGACGGTTGG $-3^{\prime}$ and $5^{\prime}$ - CGGCAGTAGGAATACAGAAGC $-3^{\prime}$; and GAPDH, 5'- TGA AGG TCG GAG TCA ACG GAT TTG GT $-3^{\prime}$ and $5^{\prime}$ - CAT GTG GGC CAT GAG GTC CAC CAC $-3^{\prime}$.

\section{Confocal microscopy}

Cells were washed with PBS, fixed in $4 \%$ paraformaldehyde for $10 \mathrm{~min}$ and permeabilized in $0.5 \%$ Triton X-100 for $10 \mathrm{~min}$ at RT. The cells were blocked and incubated with primary antibodies and then FITC-conjugated secondary antibodies. The cells were washed three times with PBS and stained with Hoechst for $3 \sim 5 \mathrm{~min}$ before imaged with a TCS-SP laser scanning confocal microscope with a $63 \times$ oil immersion lens (Leica Microsystems, Mannheim, Germany).

\section{Cell proliferation assays}

CCK8 proliferation assays (Dojindo Molecular Technologies, Japan) were performed to detect the function of SZRD1 on proliferation. 3000 or 5000 transfected cells were seeded in 96-well plates, and then cultured at $37^{\circ} \mathrm{C}$ in a $5 \% \mathrm{CO}_{2}$ humidified atmosphere. At detected time points, each well was added $10 \mu \mathrm{L}$ CCK-8 solution and incubated for $2-4 \mathrm{~h}$ at $37^{\circ} \mathrm{C}$. To calculate the number of viable cells, the staining intensity was measured using the absorbance at 450 and $630 \mathrm{~nm}$. The results were presented as the means \pm standard deviation (SD). The results were based on three independent experiments performed at three different times.

\section{Colony formation assay}

Colony formation was analyzed by plating 600cells in 6-well culture plates. After 14days, the colonies were fixed in $4 \%$ paraformaldehyde for $10 \mathrm{~min}$, and then stained with $0.5 \%$ crystal violet for $10 \mathrm{~min}$ at RT. A colony contained at least 50cells. The number of colonies was counted using image analysis software (IPP6). The results were based on three independent experiments performed at three different times.

\section{Cell cycle analysis}

The transfected cells were harvested at $24 \mathrm{~h}$ and 48h, then washed with PBS for three times. Using cold $70 \%$ ethanol fixed the cells overnight at $-20^{\circ} \mathrm{C}$, and on the second day fixed cells were collected by centrifugation, washed three times in PBS, and followed by 10ul RNaseA (Sigma-Aldrich) in PBS with $30 \mathrm{~min}$ incubation at $37^{\circ} \mathrm{C}$. At last stained with $10 \mathrm{mg} / \mathrm{mL}$ PI (Sigma-Aldrich) in 0.1\%Triton X-100. All stained cells were subjected to BD FACS Calibur (BD Bioscience, San Jose, CA, USA). The cell cycle distribution was analyzed using the ModFit LT software (Verity Software House, Topsham, ME).

\section{Cell apoptosis assay}

HeLa cells were transfected with PLEX-null or PLEX-SZRD1 plasmids. After $48 \mathrm{~h}$, the transfected cells $\left(5^{*} 10^{5}\right)$ were collected, washed twice with PBS and resuspended in $100 \mathrm{ul}$ of binding buffer $(10 \mathrm{mM}$ HEPES, pH7.4; $140 \mathrm{mM} \mathrm{NaCl} ; 1 \mathrm{mM} \mathrm{MgCl} 2 ; 5 \mathrm{mM} \mathrm{KCl}$; and $2.5 \mathrm{mM} \mathrm{CaCl} 2$ ). FITC-conjugated Annexin V was added at a final concentration of $0.5 \mathrm{ug} / \mathrm{ml}$, and the cells were further incubated for $30 \mathrm{~min}$ in ice box avoided light. After washed twice, the samples were treated with PI and analyzed on a BD FACS Calibur (BD Bioscience, San Jose, CA, USA).

\section{Western blot analysis}

The cells were re-suspended in RIPA buffer (Sigma-Aldrich, St. Louis, MO, USA) and lysised for $30 \mathrm{~min}$ in the ice box. Protein concentrations were measured according to the BCA protein assays (Pierce, Rockford, IL, USA). The whole cell lysates were performed on $12.5 \%$ or $15 \%$ SDS-PAGE gels to separate the target protein and transferred to polyvinylidene difluoride membranes (Hybond; GE Healthcare, Buckinghamshire, United Kingdom). The membrane was probed with primary antibodies overnight at $4^{\circ} \mathrm{C}$. The membrane was washed with TBST and incubated with HRP-labeled secondary antibodies. $\beta$-actin was used as a loading control.

\section{Tissue microarray (TMA) analysis}

Cervical squamous cancer samples (paraffin embedded cancer tissue blocks; $\mathrm{n}=31$ ) were purchased from the Shanghai Outdo Biotech Co., Ltd (Shanghai, China). These samples were obtained from the hospital after ethical approval. Tissue microarrays 
(TMA) were deparaffinized, and then rehydrated with ethanol. Endogenous peroxidase was quenched by $3 \% \mathrm{H}_{2} \mathrm{O}_{2}$. The slides were blocked using $10 \%$ normal goat serum for $30 \mathrm{~min}$ at room temperature and incubated with anti-SZRD1 polyclonal antibody at a 1:100 dilution overnight at $4^{\circ} \mathrm{C}$.

\section{Statistical analysis}

Student's $t$ test was used to analyze the results, expressed as the means \pm SD. $\mathrm{P}$ value $<0.05$ was considered to be a statistically significant difference.

\section{Results}

\section{Bioinformatics analysis of SZRD 1}

The gene SZRD1 is located on chromosomes 1p36.13 and comprises 4 exons and 3 introns. SZRD1 encodes a highly conserved protein in Homo sapiens, Pan troglodytes, Macaca mulatta, Bos Taurus, Mus musculus, Rattus norvegicus, Canis lupus familiaris, Gallus gallus, Xenopus tropicalis and Danio rerio (Figure 1A). The amino acid sequence of Homo sapiens, Pan troglodytes and Macaca mulatta are exactly identical, and their homology are as high as $100 \%$. The SignalP software (http://www. cbs.dtu.dk/services/SignalP/) analysis predicted that SZRD1 didn't contain potential signal peptide, The
TMHMM software (http://www.cbs.dtu.dk/ services/TMHMM/) analysis predicted that SZRD1 didn't contain transmembrane region. These data suggest that SZRD1 is a highly conserved intracellular protein.

\section{Expression pattern and localization in cells of SZRD 1}

The expression of SZRD1 in normal human tissues and cell lines were detected by real-time PCR, we found that SZRD1 was expressed in a wide spectrum of tissues, and with high levels of expression detected in leukocyte, lung, lymph node, pancreas, placenta and spleen. In various cell lines, the SZRD1 expression levels were detected and showed a higher expression in Jurkat, K562, PANC1, Raji, THP1 and U937 cell lines compared with others (Figure 1B). Using SZRD1 specific polyclonal antibody prepared in our laboratory, the localization of SZRD1 in HeLa cells was detected by confocal microscopy. The results showed that SZRD1 localized to the cytoplasm and nucleus but excluded from the nucleoli (Figure 1C), and the results is further supported by protein atlas (http://www. proteinatlas.org/ENSG00000055070-SZRD1/subcellu lar) databases in U2OS cell line.
A

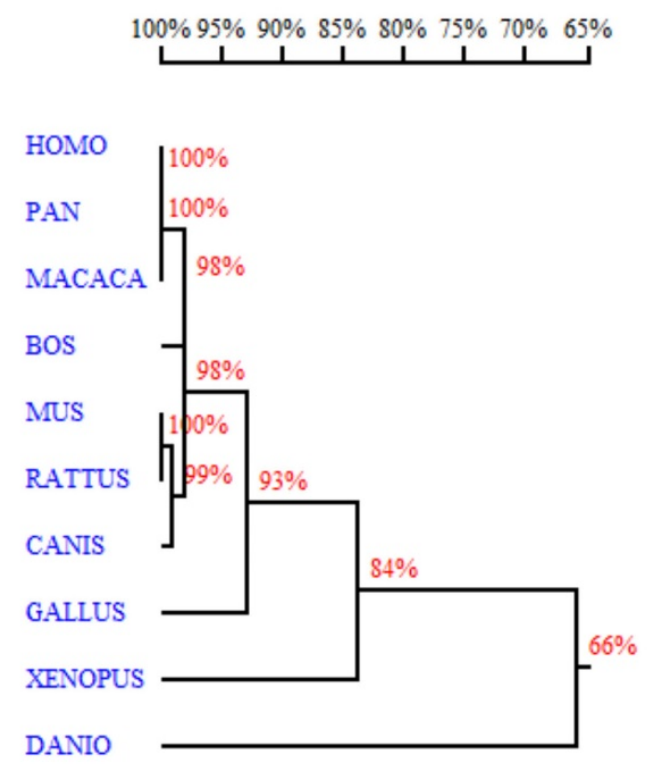

B

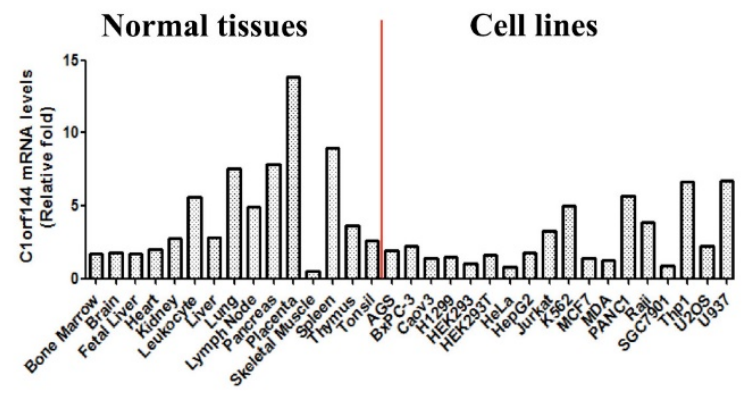

C

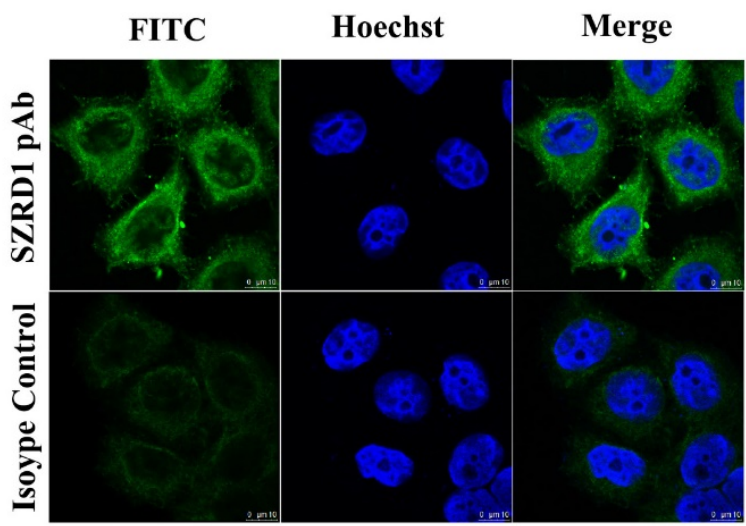

Figure 1. Bioinformatics analysis, expression pattern and localization in cells of SZRD1. A: Homology tree of SZRD1 from different species. The similarity of the amino acid sequences between different species was indicated by percentage. B: Expression pattern of SZRDI was detected using real-time qPCR in a panel of normal human tissues and cell lines. C: Endogenous localization of SZRD1 in HeLa Cells. 
A

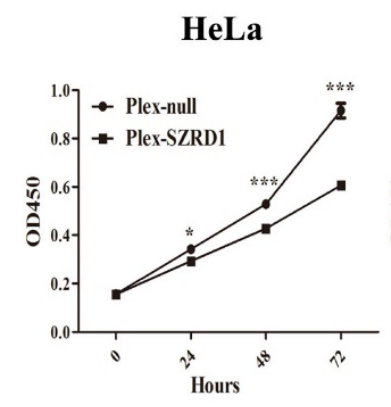

$293 T$

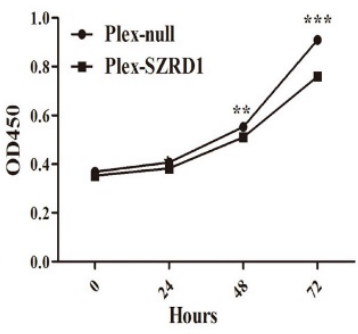

B

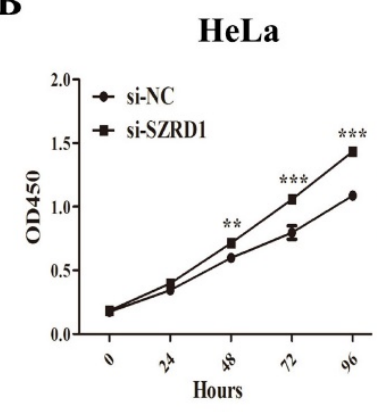

K562

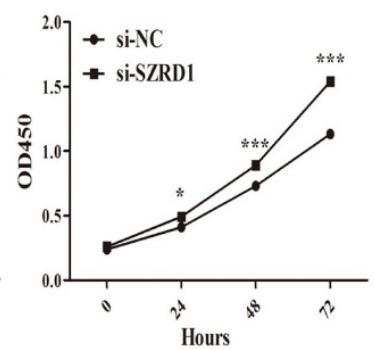

C
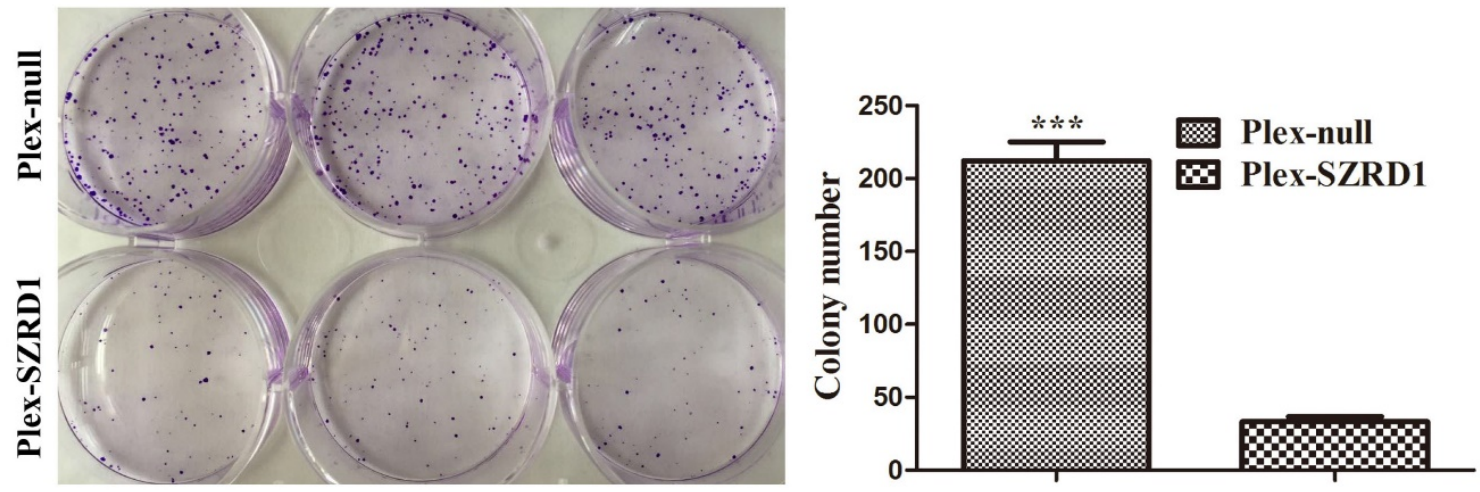

Figure 2. SZRDI can inhibit cell proliferation in vitro. A: The Cell Counting Kit-8 assays were performed to determine the proliferation of HeLa and 239T cells transfected with SZRDI plasmid or control. The results are expressed as the means \pm SEM of three independent experiments performed at three different times. *, $\mathrm{P}<0.05 ; * *, \mathrm{P}<0.01$; and ***, $\mathrm{P}<0.001$ compared to the controls. B: The Cell Counting Kit-8 assays were performed to determine the proliferation of HeLa and K562 cells transfected with si-SZRDI or si-NC. Si-NC, negative control siRNA. The results are expressed as the means \pm SEM of three independent experiments performed at three different times. C: $600 \mathrm{HeLa}$ cells transfected with SZRDI or control plasmids were cultured routinely in a 6 well plate for 14 days. Results are presented for triplicate wells.

\section{SZRDI can suppress cells proliferation in vitro}

To investigate the function of SZRD1, we analyzed the correlated genes in HEK293 cells by using 228 samples downloaded from the GEO database. David enrichment analysis revealed that the most-correlated genes to SZRD1 were associated with cell cycle (GO:007049 and GO:0022402), suggesting a potential role of the gene in cell cycle regulation. Similar GO terms relevant to cell cycle such as DNA replication (GO:0006260), DNA biosynthetic process (GO:0071897) and cell cycle checkpoint (GO:0000075) were also observed to be enriched among the correlated genes of SZRD1 if we used human HEK293T cells (129 samples) to perform correlation analysis. This functional clue was further verified by experiments.

SZRD1 transfected tumor cells HeLa and normal cells 293T displayed significantly decreased cell viability by CCK-8 assay compared to cells transfected with the negative controls (Figure 2A). In contrast, the viability of HeLa and K562 cells increased when endogenous SZRD1 was knocked down with si-SZRD1 (Figure 2B). We further assessed the effect of SZRD1 on the proliferation of HeLa cells using colony formation assay. SZRD1 restrained colony formation compared with the control group in HeLa cells (Figure 2C).

\section{SZRDI induces cell cycle arrest and apoptosis in HeLa cells}

We observed strong growth inhibition of several cell lines after transfected SZRD1 plasmids. To further detect the mechanism, we continued to examine the effect of SZRD1 on the cell cycle distribution of HeLa using PI staining assay. As shown in (Figure 3A), after transfected HeLa cells 48 hours, there was a significant increase in G2/M phase in SZRD1 transfected cells compared to cells transfected with negative controls. To explore the mechanism underlying the cell cycle arrest induced by SZRD1, we measured the regulators that involved cell cycle progress. The CDK inhibitor P21, which participates in G2 phase regulation, was upregulated by SZRD1 (Figure 3C). On the other hand, the endogenous protein of SZRD1 was knocked down with si-SZRD1 in HeLa cells (Figure 3B), then the expression of P21 was downregulated, which was consistent with the results of overexpression (Figure 3C).

Furthermore, apoptosis assay using Annexin $\mathrm{V} / \mathrm{PI}$ staining showed that the percentage of apoptotic cells was significantly higher in SZRD1 transfected 
HeLa cells compared to cells transfected with negative controls (Figure 3D). The expression of apoptosis related caspase 3 protein was detected. SZRD1 induced the cleavage of caspase-3, which suggested that it induces apoptosis through activating the caspase family protein (Figure 3E). Taken together, our findings suggest that SZRD1 can induce cell cycle arrest and apoptosis in HeLa cells.

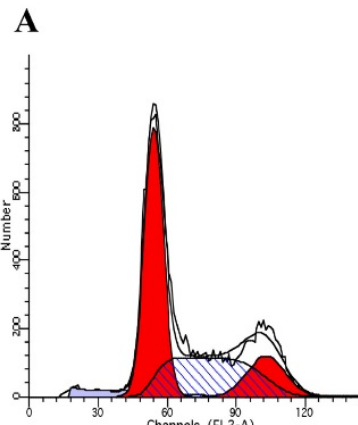

Plex-null(24h)

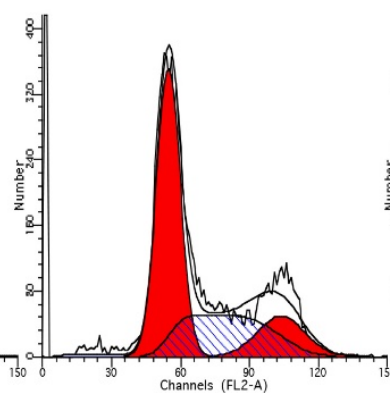

Plex-SZRD1(24h)

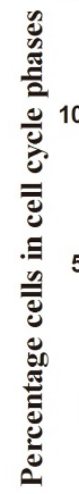

D

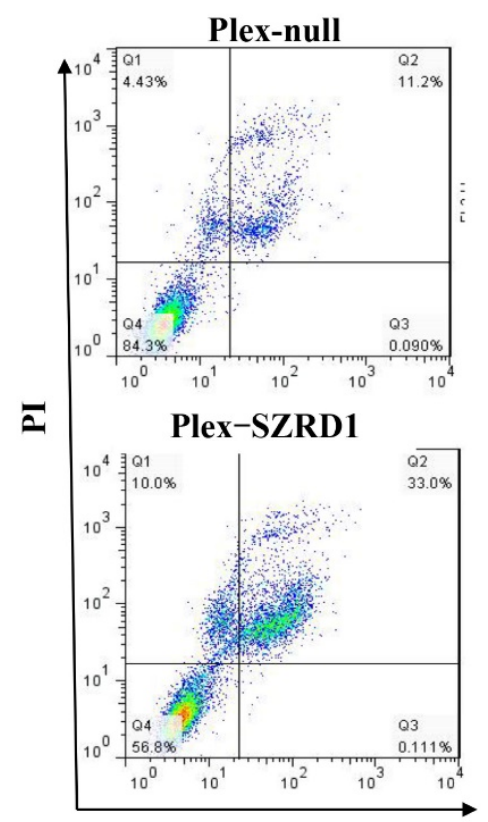

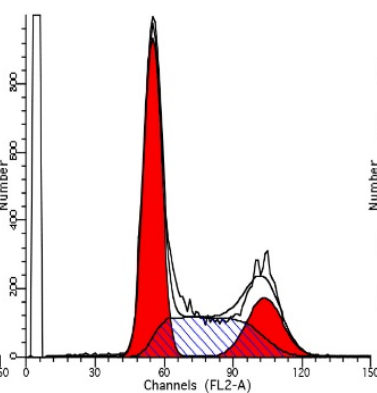

Plex-null(48h)

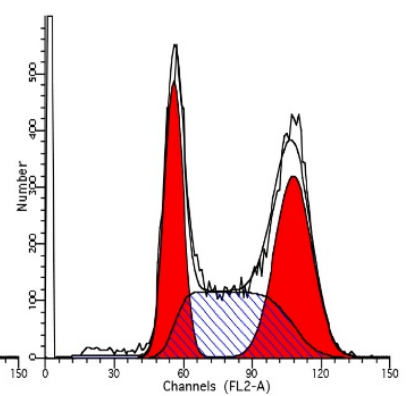

Plex-SZRD1(48h)

B
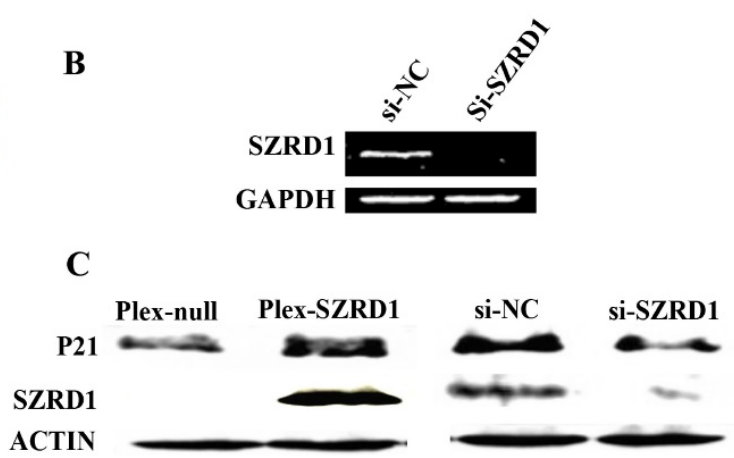

E

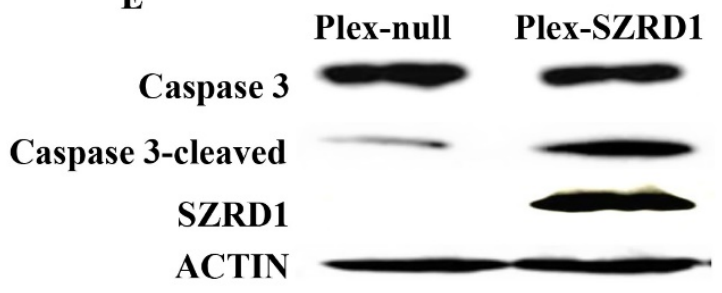

FITC-Annexin V

Figure 3. SZRDI induces cell cycle arrest and apoptosis in HeLa cells. A: The cell cycle was analyzed $24 \mathrm{~h}$ and $48 \mathrm{~h}$ after transfection of HeLa cells by flow cytometry. The percentage of cells at different phases (lower panel) is shown. B: Knockdown of SZRDI with siRNA in HeLa cells was verified by semi-quantitative RT-PCR. C: The expression of P21 was evaluated in HeLa cells $48 \mathrm{~h}$ transfected with SZRD1 plasmid or si-SZRD1 using western blotting. D: Annexin V/PI-staining was performed to analyze apoptosis rate of HeLa cells $48 \mathrm{~h}$ after transfected with SZRD1 plasmid. Picture is a representative result of three independent experiments. $* * *, P<0.001$. E: The expression of caspase 3 was evaluated in HeLa cells $48 \mathrm{~h}$ transfected with SZRDI plasmid using western blotting. 


\section{SZRD1 inhibits cell proliferation by suppressing phosphorylation of ERK1/2, AKT and STAT3 and its downstream effectors}

To further investigate the mechanism responsible for the effect of SZRD1 on cell proliferation and apoptosis rate, the related protein levels were evaluated using western blotting analysis. Western blotting analysis demonstrated the phosphorylation of ERK1/2, AKT and STAT3 were suppressed by SZRD1(Figure 4A). On the other hand, ERK1/2, AKT and STAT3 were activated after SZRD1 knockdown (Figure 4B). These results correlated with the changes of RAF1, RAS, mTOR and P70 (Figures $4 \mathrm{~A}$ and $4 \mathrm{~B})$. ERK1/2 activation is generally associated with enhanced cell survival, and blocking the RAF1/RAS/ERK1/2 pathway is widely used as an antineoplastic method. In our study, SZRD1 the other hand, SZRD1 treatment had little effect on phosphorylated p38 and JNK. It could be concluded that SZRD1 suppresses the ERK1/2, AKT, STAT3 pathway and its downstream effectors, and further inhibits cells proliferation and promote apoptosis.

\section{SZRD1 is downregulated in many cancers according to the omic data analysis}

Gene expression profiles in multiple cancers and their corresponding control (normal or non-tumour) tissues can provide clues to the function of it. In order significantly abrogated ERK1/2 phosphorylation. On

to study the possibility of SZRD1 as a tumor suppressor, the differential expression of SZRD1 in cancers and corresponding control at the mRNA level were analyzed by an integrated bioinformatics analysis based on the omic tumour data. The average rank scores (ARS) denoted the level of SZRD1 expression in multiple cancers (Table 1). The P-values were calculated using the Wilcoxon rank-sum test in the R (http://www.r-project.org/) software environment and adjusted by the ARS and bonferroni correction. The smaller P-value was, the greater SZRD1 expression difference between the cancer tissues and the corresponding normal tissues would be. Analysis showed a downregulation of SZRD1 in many cancers, such as uterus endometrium cancer, breast cancer, brain neuroblastoma, gastric cancer and rectum adenocarcinoma, etc, while no apparent differences were detected in bladder cancer, kidney ccRCC, retinoblastoma, etc. Only in lymphoma MCL, SZRD1 was significantly upregulated. The result is also supported by the BioXpress (http://hive.bio chemistry.gwu.edu/tools/bioxpress).

\section{Downregulation of SZRDI expression is associated with progression of human cervical cancer}

To determine the clinical relevance of SZRD1 expression in patients suffered from cervical cancer, TMA of cervical squamous cancer was analyzed to evaluate the expression of SZRD1. Immunohistochemical analysis showed that the protein levels of
A

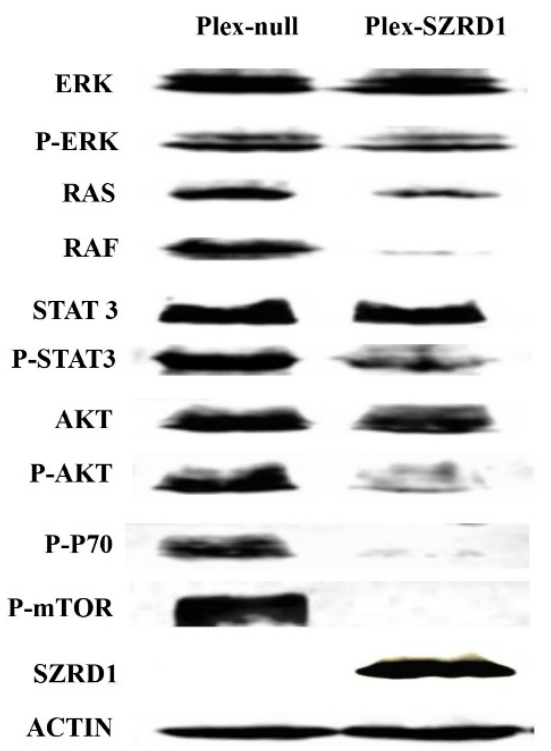

B

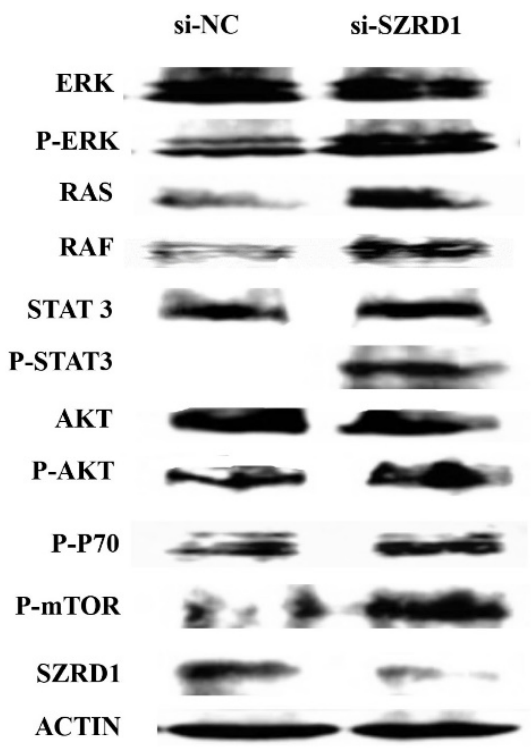

Figure 4. SZRD1 inhibits cell proliferation by suppressing phosphorylation of ERK, AKT and STAT3 and its downstream effectors. A: Effect of SZRDI on the phosphorylation of ERK, AKT and STAT3 in HeLa cells. Cells were transfected with SZRDI plasmid for $48 \mathrm{~h}$. The phosphorylation of ERK, AKT and STAT3 were evaluated using western blotting. B: Effect of SZRD1 knockdown on the phosphorylation of ERK, AKT and STAT3 regulators in HeLa cells. Cells were transfected with si-SZRDI or si-NC for $48 \mathrm{~h}$. The phosphorylation of ERK, AKT and STAT3 were evaluated using western blotting.
SZRD1 were significantly downregulated in $26(83.9 \%)$ cervical squamous cancer samples compared with adjacent normal cervical squamous epithelium tissues (Figure 5A). Furthermore, compared with corresponding normal tissue, the amount of the sample with low SZRD1 expression accounts for a higher percentage in the high stage malignant cervical cancer, which indicated that SZRD1 expression was negatively correlated with clinical stages (Figure 5B). The results of SZRD1 expression are consistent with omic data analysis in patients suffered from cervical cancer. 
Table 1. The average expression levels of SZRD1 in multiple cancers.

\begin{tabular}{|c|c|c|c|c|}
\hline Tissues & Sample & $\mathrm{ARS}^{\mathbf{a}}$ & P value ${ }^{b}$ & Bonferronic \\
\hline Lung NSCL & 582 & 82.76 & 0.7798954 & 1 \\
\hline Lung adenocarcinoma & 1128 & 81.18 & $2.78 \mathrm{E}-08$ & 0.0015028 \\
\hline Lung nonTumour & 470 & 82.70 & & \\
\hline$\underline{\text { Breast cancer }^{d}}$ & 2689 & 81.48 & 2.89E-20 & $1.58 \mathrm{E}-15$ \\
\hline Breast IDCd & 822 & 81.28 & $1.56 \mathrm{E}-16$ & 8.52E-12 \\
\hline Breast TNd & 274 & 80.73 & $9.84 \mathrm{E}-18$ & 5.37E-13 \\
\hline Breast nonTumour & 234 & 84.50 & & \\
\hline Brain glioblastoma & 668 & 80.53 & 0.2269274 & 1 \\
\hline Brain meningioma & 89 & 83.56 & 3.74E-11 & 2.04E-06 \\
\hline Brain oligodendroglioma & 151 & 79.73 & 0.0161423 & 1 \\
\hline$\underline{\text { Brain neuroblastomad }}$ & 255 & 73.90 & $1.73 \mathrm{E}-26$ & $9.44 \mathrm{E}-22$ \\
\hline Brain astrocytoma pilocytic & 156 & 76.89 & 2.90E14 & $1.58 \mathrm{E}-09$ \\
\hline Brain ependymoma & 371 & 78.76 & 3.81E07 & 0.0207785 \\
\hline$\underline{B r a i n}$ medulloblastomad $^{\text {din }}$ & 353 & 74.48 & 4.47E-27 & $2.44 \mathrm{E}-22$ \\
\hline Brain nonTumour & 122 & 80.91 & & \\
\hline Uterus endometrium cancerd & 371 & 79.51 & 7.14E-15 & $3.90 \mathrm{E}-10$ \\
\hline $\begin{array}{l}\text { Uterus endometrium } \\
\text { nonTumour }\end{array}$ & 421 & 84.25 & & \\
\hline Uterus cervical cancer & 241 & 84.64 & 0.0177781 & 1 \\
\hline Uterus nonTumour & 41 & 87 & & \\
\hline Lymphoma MCL & 78 & 85.69 & 2.28E-12 & $1.24 \mathrm{E}-07$ \\
\hline Lymphoma DLBCL GCB & 585 & 82.11 & 3.97E-10 & 2.17E-05 \\
\hline Lymph node nonTumour & 65 & 78.25 & & \\
\hline Gastric cancerd & 925 & 82.56 & $2.55 \mathrm{E}-13$ & 1.39E-08 \\
\hline Gastric nonTumour & 93 & 86.20 & & \\
\hline Kidney ccRCC & 651 & 82.85 & 0.0114607 & 1 \\
\hline Kidney nonTumour & 244 & 82.27 & & \\
\hline Liver HCC & 383 & 78.30 & 0.0061783 & 1 \\
\hline Liver nonTumour & 497 & 79.03 & & \\
\hline Retinoblastoma & 78 & 73.50 & 0.7171119 & 1 \\
\hline Retina nonTumour & 50 & 74.58 & & \\
\hline Bladder cancer & 186 & 82.73 & 0.291811 & 1 \\
\hline Bladder nonTumour & 64 & 81.73 & & \\
\hline Colon cancer & 470 & 83.35 & $1.34 \mathrm{E}-07$ & 0.0072912 \\
\hline Colon mucosa nonTumour & 475 & 84.99 & & \\
\hline Colorectal adenocarcinoma & 977 & 82.81 & $1.60 \mathrm{E}-07$ & 0.0087467 \\
\hline Colorectal tissue nonTumour & 99 & 85.11 & & \\
\hline$\underline{\text { Rectum adenocarcinomad }^{\mathrm{d}}}$ & 104 & 83.47 & 8.47E-29 & 4.61E-24 \\
\hline Rectum mucosa nonTumour & 221 & 88.91 & & \\
\hline Prostate cancer & 298 & 82.11 & 1.67E-07 & 0.0091217 \\
\hline Prostate nonTumour & 102 & 84.99 & & \\
\hline Ovarian cancer & 231 & 84.69 & 0.2204898 & 1 \\
\hline Ovarian endometrioid cancer & 121 & 81.98 & 0.0051993 & 1 \\
\hline Ovarian cancer serous & 999 & 82.37 & 0.0193773 & 1 \\
\hline $\begin{array}{l}\text { Ovarian epithelium } \\
\text { nonTumour }\end{array}$ & 90 & 84.48 & & \\
\hline
\end{tabular}

aARS denotes the average rank score.

bThe P-values were calculated using the Wilcoxon rank-sum test in the $\mathrm{R}$ (http://www.r-project.org/) software environment and are relative to the corresponding non-tumor or normal tissues.

cThe P-values were adjusted using Bonferroni correction in the function "p.adjust" in the R software.

dThe expression intensities of SZRD1 in the underlined tissues were considered to be significantly downregulated by fully considering the differences between the ARS values.

\section{Discussion}

With the discovery of oncogenes and tumor suppressor genes, there is a great improvement in understanding of tumor mechanism. Researchers are committed to make great efforts to study the function of new genes. SZRD1 is a novel gene without any systematic functional reports currently. Homology analysis shows that it is a highly conserved protein, and have homology with 180 species of organisms. SZRD1 contains three conserved regions of $\mathrm{N}$-terminal, SUZ and SUZ-C domain, and have similar structure with SZY-20, which localizes to the centrosome and negatively regulates centrosome duplication [8]. These indicate that SZRD1 may play a very important role in the evolution of biological, especially in the cell cycle. It is of great significance to make clear the function of SZRD1.
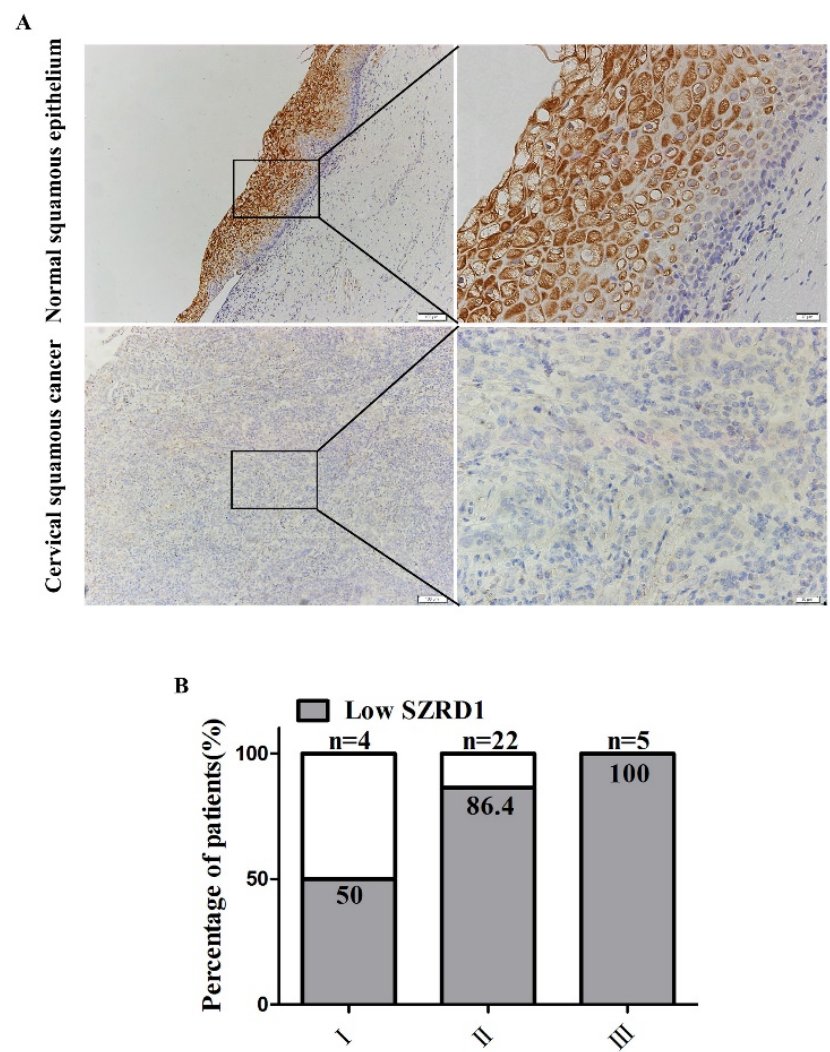

Figure 5. SZRDI is downregulated in cervical squamous cancer. A: Representative pictures of SZRD1 expression in cervical squamous cancer tissue and normal tissue. TMA of human cervical squamous cancer patient tissues (total $n=31$ ). Magnification, $\times 100$ and $\times 400$ as indicated. The slides were stained with anti-SZRD1 antibody. B: Percentage of patients with low expression of SZRDI according to different clinical stage. Lower expression refers to the level of SZRDI in cancer is lower than the corresponding normal tissue.

In order to study the biological function of SZRD1, we conducted a bioinformatics analysis. The results also showed that SZRD1 played an important role in cell cycle, which was consistent with our previous speculation and had been verified in subsequent research. The results of the study demonstrated that overexpression of SZRD1 inhibited cell proliferation and induced cell apoptosis through increased G2/M phase cell cycle arrest, while epigenetic silencing of SZRD1 increased cell viability. We found that overexpression of SZRD1 inhibited cell proliferation and induced cell apoptosis accompanied 
by significant G2/M arrest. The cell cycle is a normal process of eukaryotic cell division. When the exogenous stimulation of cells causes DNA damage, the cells are arrested in the G1 or G2 phase until the damage is repaired. Mitosis entry is regulated by cell division cycle (cdc)/cyclin B1 complexes. Cdc2, the cyclin dependent kinase that normally drives cells into mitosis, is the ultimate target of pathways that mediate rapid arrest in G2 in response to DNA damage [19]. The P21 protein directly blocks cell cycle progression by inhibiting the activity of cyclin B1/cdc2 complexes that normally promote G2/M phase progression $[9,20]$. In the present study, the expression of P21 can be upregulated by SZRD1 and are markedly decreased when knockdown of the SZRD1. Therefore, these results support the view that SZRD1 induces cell cycle arrest at G2/M phase through regulating P21.

Apoptosis is a spontaneous and programmed process of cell death under normal physiological or pathological conditions. Apoptosis pathways converge on activation of procaspase 3 which in turn cleaves PARP and activates endonucleases leading to DNA fragmentation [21]. The results showed that the apoptosis rates of HeLa cell transfected SZRD1 plasmid was increased using flow cytometry analysis. Additionally, western blotting results showed that caspase 3 was cleaved in HeLa cell transfected SZRD1 plasmid, which demonstrated the occurrence of cell apoptosis.

Further investigation of signaling pathways in HeLa revealed that overexpression of SZRD1 caused dramatic decrease in ERK1/2, AKT and STAT3 phosphorylation and had no or little effect on p38 phosphorylation. MAPKs and AKT are fundamental survival pathways activated and regarded as important targets of clinical chemotherapeutic drugs [22]. These two pathways promote cell survival by phosphorylating substrates that decrease the activity of pro-apoptotic proteins or increase the activity of anti-apoptotic proteins [23]. Phosphorylation of the key cytoprotective molecules ERK1/2 and AKT were substantially reduced which resulted in significant suppression of HeLa and 293T cell proliferation. Aside from promotion of cell growth, ERK1/2 protein kinases may act directly or indirectly on cyclins to arrest division and permit differentiation [12]. ERK1/2 deactivation may be linked to apoptosis induction, given that the inhibition of ERK1/2 is primarily required to induce apoptosis [13]. Moreover, other signaling molecules also play crucial roles in the pathway of SZRD1. For example, activation of the JAK/STAT3 pathway also contributes to the initiating of diseases [24, 25], and therefore JAK/STAT3 has been considered as an attractive therapeutic target for developing therapeutic agents. The results obtained from the present study suggested that the ERK1/2, AKT and STAT3 pathways are the targets for SZRD1 in HeLa cells.

SZRD1 plays an important role in the cell cycle. It can inhibit cell proliferation and induce apoptosis in G2 / M phase in HeLa, so it may be a tumor suppressor gene in cervical cancer. Cervical cancer is one of the most common gynecological malignancies, and in recent years its incidence has a rising trend in some countries and regions. However, there isn't any report about the expression of SZRD1 in cervical cancer. In this study, bioinformatics analysis and immunohistochemistry were carried out to detect the expression of SZRD1 in cervical squamous cancer. The results showed that SZRD1 expression was frequently downregulated in cervical cancer tissues compared with adjacent normal cervical squamous epithelium tissues. Moreover, the clinical significance of SZRD1 in cervical cancer progression was determined, as its expression was negatively correlated with malignant phenotypes of cervical cancer.

\section{Conclusions}

In summary, this is the first time systematic research on SZRD1 has been carried out and the first time the function of SZRD1 has been reported in the world. This study shows that the basic function of SZRD1 is inhibiting cell proliferation and inducing cell apoptosis. The expression of SZRD1 is frequently reduced in cervical cancer tissues according to omic data analysis as well as our immunohistochemistry experimental data, suggesting that SZRD1 functions as a tumor suppressor in cervical cancer and may play an important role in preventing tumorigenesis and progression. These findings not only provide insights into the underlying molecular mechanism of SZRD1 in cervical cancer, but also have great significance to the diagnosis and treatment of cervical cancer.

\section{Acknowledgments}

This work was supported by grants from the National Natural Science Foundation of China. (NO.91542106, NO.81172001).

\section{Ethical approval}

This article does not contain any studies with human participants or animals performed by any of the authors.

\section{Competing Interests}

The authors have declared that no competing interests exist. 


\section{References}

1. Nesbit CE, Tersak JM, Prochownik EV. MYC oncogenes and human neoplastic disease. Oncogene. 1999; 18: 3004-16.

2. Bromberg JF, Wrzeszczynska MH, Devgan G, Zhao Y, Pestell RG, Albanese C, et al. Stat3 as an oncogene. Cell. 1999; 98: 295-303.

3. Soussi T, Ishioka C, Claustres M, Beroud C. Locus-specific mutation databases: pitfalls and good practice based on the p53 experience. Nature reviews Cancer. 2006; 6: 83-90

4. Wang X, Jiang X. Post-translational regulation of PTEN. Oncogene. 2008; 27: 5454-63.

5. Zhang H, Ma X, Shi T, Song Q, Zhao H, Ma D. NSA2, a novel nucleolus protein regulates cell proliferation and cell cycle. Biochemical and biophysical research communications. 2010; 391: 651-8.

6. Wang P, Sun B, Hao D, Zhang X, Shi T, Ma D. Human TMEM174 that is highly expressed in kidney tissue activates AP-1 and promotes cell proliferation. Biochemical and biophysical research communications. 2010; 394: 993-9.

7. He P, Peng Z, Luo Y, Wang L, Yu P, Deng W, et al. High-throughput functional screening for autophagy-related genes and identification of TM9SF1 as an autophagosome-inducing gene. Autophagy. 2009; 5: 52-60.

8. Song MH, Aravind L, Muller-Reichert T, O'Connell KF. The conserved protein SZY-20 opposes the Plk4-related kinase ZYG-1 to limit centrosome size. Developmental cell. 2008; 15: 901-12.

9. Bloom J, Cross FR. Multiple levels of cyclin specificity in cell-cycle control. Nature reviews Molecular cell biology. 2007; 8: 149-60.

10. Vera J, Raatz Y, Wolkenhauer O, Kottek T, Bhattacharya A, Simon JC, et al. Chk1 and Wee1 control genotoxic-stress induced G2-M arrest in melanoma cells. Cellular signalling. 2015; 27: 951-60.

11. McDonald ER, 3rd, Wu GS, Waldman T, El-Deiry WS. Repair Defect in p21 WAF1/CIP1 -/- human cancer cells. Cancer research. 1996; 56: 2250-5.

12. Galluzzi L, Aaronson SA, Abrams J, Alnemri ES, Andrews DW, Baehrecke EH, et al. Guidelines for the use and interpretation of assays for monitoring cell death in higher eukaryotes. Cell death and differentiation. 2009; 16: 1093-107.

13. Marino G, Niso-Santano M, Baehrecke EH, Kroemer G. Self-consumption: the interplay of autophagy and apoptosis. Nature reviews Molecular cell biology. 2014; 15: 81-94.

14. Chen T, Stephens PA, Middleton FK, Curtin NJ. Targeting the S and G2 checkpoint to treat cancer. Drug discovery today. 2012; 17: 194-202.

15. Matsuda A, Suzuki Y, Honda G, Muramatsu S, Matsuzaki O, Nagano Y, et al. Large-scale identification and characterization of human genes that activate NF-kappaB and MAPK signaling pathways. Oncogene. 2003; 22: 3307-18.

16. Wang P, Qi H, Song S, Li S, Huang N, Han W, et al. ImmuCo: a database of gene co-expression in immune cells. Nucleic acids research. 2015; 43: D1133-9.

17. Huang da W, Sherman BT, Lempicki RA. Systematic and integrative analysis of large gene lists using DAVID bioinformatics resources. Nature protocols. 2009; 4: 44-57.

18. Wang $\mathrm{P}$, Yang $\mathrm{Y}$, Han $\mathrm{W}$, Ma D. ImmuSort, a database on gene plasticity and electronic sorting for immune cells. Scientific reports. 2015; 5: 10370 .

19. Stark GR, Taylor WR. Control of the G2/M transition. Molecular biotechnology. 2006; 32: 227-48

20. Malumbres M, Pevarello P, Barbacid M, Bischoff JR. CDK inhibitors in cancer therapy: what is next? Trends in pharmacological sciences. 2008; 29: 16-21.

21. Elmore S. Apoptosis: a review of programmed cell death. Toxicologic pathology. 2007; 35: 495-516.

22. Zhou L, Luan H, Dong X, Li Y. Activation of the PI3K/Akt and MAPK signaling pathways antagonizes adriamycin-induced HL-60 leukemia cell apoptosis. Molecular medicine reports. 2010; 3: 641-4.

23. Bononi A, Agnoletto C, De Marchi E, Marchi S, Patergnani S, Bonora M, et al. Protein kinases and phosphatases in the control of cell fate. Enzyme research. 2011; 2011: 329098

24. Aneknan P, Kukongviriyapan V, Prawan A, Kongpetch S, Sripa B, Senggunprai L. Luteolin arrests cell cycling, induces apoptosis and inhibits the JAK/STAT3 pathway in human cholangiocarcinoma cells. Asian Pacific journal of cancer prevention: APJCP. 2014; 15: 5071-6.

25. Nair RR, Tolentino JH, Hazlehurst LA. Role of STAT3 in Transformation and Drug Resistance in CML. Frontiers in oncology. 2012; 2: 30. 